\title{
e-Loran Navigation System for Egyptian Coasts \& Maritime
}

\author{
$\{\text { Farag M. Bahr*, Ezz Eldin Abdelkawy, Samy A.Shedied }\}^{\dagger}$
}

\begin{abstract}
Ship navigation demands position fixing with a high degree of accuracy to enter the harbor mariner. The International Maritime Organization (IMO) has set a list of requirements which must be met by any electronic positioning system to be used on ships. The Global Positioning System (GPS) is most often the primary source of Position, Navigation and Timing (PNT) information. Due to the low levels of GPS signal strength at the surface of the earth and low jamming immunity, the recent concerns about vulnerability of GPS has sparked a renewed interest in the Loran PNT system. Enhanced Loran or simply "eLoran" is the latest version of Loran PNT for longstanding and proven series of low frequency navigation systems which are used to provide back-up capabilities to GPS in maritime navigation.

In this paper, we present a detailed proposal study to establish eLoran navigation system for Egyptian maritime harbors. This study uses the performance requirements for both nonprecision approach (NPA) and maritime harbor entrance approach (HEA). The primary requirements of concern for NPA or HEA are the accuracy, integrity, availability and continuity. In the maritime sector, accuracy requirements are the most stringent and are considered the greatest challenge for eLoran establishment. Mitigating the sources of variation and error on Loran signal guided the design of eLoran. The simulation results for this proposal of Egyptian eLoran achieved the IMO's accuracy requirement of better than 20m.
\end{abstract}

\section{Introduction}

GPS is widely used on ships as a primary navigation system. However, as GPS has become increasingly important, the vulnerability of GPS has also become a critical issue to provide an alternative positioning system during GPS outages [1]. Recently, eLoran is considered as the most promising backup for GPS in the case of denied GPS signal. eLoran is a terrestrial lowfrequency system organized as chains each consisting of a master station with two or more secondary stations. Each station broadcasts in a strict time format, series of pulses with effective radiated power up to $4000 \mathrm{~kW}$ depending on the required working range. eLoran meets international performance standards that allow it to serve as a backup to GPS in a great number of applications across multiple sectors. The primary requirements of concern for NPA or HEA are the accuracy, integrity, availability and continuity. These requirements for NPA and HEA are seen in Table-1 [2]. There are many eLoran chains around the world including United Kingdom, USA, Saudi Arabia, India and South Korea. In this paper, we introduce a proposal to establish eLoran in the maritime transportation sector for Egyptian coasts and harbors.

The rest of the paper is organized as follows: In section 2, we define the coverage area and performance models used to establish eloran. Definition of accuracy parameter and how to get position solution are given in section 3 . In section 4 , we discuss the factors that effect on the

farag_bahr@yahoo.com

Egyptian Armed Forces, Egypt. 
position accuracy according to General Lighthouse Authorities (GLA) model. Methods used for eLoran accuracy computation are depicted in section 5. Experimental results, computations and assumptions for eLoran in Egyptian coasts and harbors are explained in section 6 . Conclusion and future work are presented in section 7.

Table 1: Primary requirements for eLoran according to NPA and HPA

\begin{tabular}{l|c|c}
\hline \hline Performance Requirement & NPA & HPA \\
\hline \hline 2DRMS accuracy (m) & 307 & 20 \\
\hline Monitor Limit/ Alert Limit (m) & 556 & 50 \\
\hline Integrity / hour & $10^{-7}$ & $3 \times 10^{-5}$ \\
\hline Time-to-Alert (sec) & 10 & 10 \\
\hline Availability & 99.9 to $99.99 \%$ & $99.7 \%$ \\
\hline Continuity & 99.9 to 99.99 & $99.85 \%$ over 3 hours \\
\hline Frequency Accuracy & $10^{-11}$ & $10^{-11}$ \\
\hline Time Accuracy (ns) & 50 & 50 \\
\hline \hline
\end{tabular}

\section{2. eLoran Coverage Area and Performance Models}

The coverage of a positioning system is the result of four performance factors including accuracy, availability, continuity and integrity [3]. eLoran coverage prediction has been performed for many years where standard models are introduced to describe the coverage and performance requirements to establish eLoran system.: (1) USCG Loran-C accuracy model, (2) Bangor model to meet the needs of the North-West European Loran Systems, (3) Loran Integrity Performance Panel (LORIPP)LLoran Accuracy Performance Panel (LORAPP) models and finally (4) GLA model. Table-2 gives a summary of establishing parameters which are considered in each model [3].

Table 2: Performance models for eLoran establishment

\begin{tabular}{|c|c|c|c|c|}
\hline \multirow{2}{*}{ Model Parameter } & \multicolumn{4}{|c|}{ Performance Model } \\
\hline & USCG & Bangor & LORIPP\LORAPP & GLA \\
\hline Station Range SNR $\geq-10 \mathrm{~dB}$ & $\sqrt{1}$ & $\sqrt{1}$ & $\sqrt{1}$ & $\sqrt{1}$ \\
\hline Geometrical Fix Accuracy & $\sqrt{ }$ & $\sqrt{ }$ & $\sqrt{ }$ & $\sqrt{ }$ \\
\hline Sky Wave Interference & & $\sqrt{ }$ & $\sqrt{ }$ & $\sqrt{ }$ \\
\hline Envelop Coding Delay & & $\sqrt{ }$ & $\sqrt{ }$ & $\sqrt{ }$ \\
\hline Continuous-Wave Interference & & $\sqrt{ }$ & $\sqrt{ }$ & $\sqrt{ }$ \\
\hline ASF Differential Correction & & & $\sqrt{ }$ & $\sqrt{ }$ \\
\hline Availability & & & $\sqrt{ }$ & $\sqrt{ }$ \\
\hline Continuity & & & $\sqrt{ }$ & $\sqrt{ }$ \\
\hline Coverage Region Divided Grids & & & & $\sqrt{ }$ \\
\hline
\end{tabular}

\section{Accuracy of eLoran and Position Solution}

The accuracy of any positioning system is defined as the closeness of its navigational solution to the true position. Position accuracy can be divided into two types: (1) absolute accuracy which is measured with respect to the geographic coordinates of the earth and (2) repeatable accuracy which is measured by how the user can return to a position whose coordinates have been measured at a previous time with the same navigational system. In this paper, we explain how eLoran absolute accuracy can be enhanced to the level of its repeatable accuracy and we present a method for evaluation of eLoran repeatable accuracy. As eLoran is a ranging system, the pseudo range $\rho$ from the receiver to every eLoran transmitter can be represented as: 


$$
\rho=\mathrm{R}+\mathrm{PF}+\mathrm{SF}+\mathrm{ASF}+\delta+\varepsilon+\mathrm{b}
$$

where:

$\mathrm{R}=$ the true range, $\mathrm{PF}=$ primary factor, $\mathrm{SF}=$ secondary factor, $\mathrm{ASF}=$ additional secondary factor, $\delta=$ variation in $\mathrm{PF}, \mathrm{SF}$ and $\mathrm{ASF}, \varepsilon=$ remaining measurement errors, $\mathrm{b}=$ the receiver clock bias

We have assumed that eLoran position-fixing is derived using an over-determined LeastSquares solution of pseudorange observations. Since ground wave geodetic range measurements are not linearly related to position, we linearize the pseudo range equation, at a nominal position $\left(\mathrm{x}_{\circ}, \mathrm{y}_{\mathrm{o}}\right)$ to give the linear matrix equation given by:

$$
\Delta \rho=A . \Delta p
$$

where $\mathrm{A}$ is the direction cosine matrix or the geometry matrix and the correction vector $\Delta \mathrm{p}$ is then used to update the position solution estimate. $\Delta \rho$ is the vector of corresponding changes in the measured pseudo range relative to its nominal value $\rho$ (Хо, уо).

The equation (2) can be expanded to:

$$
\Delta \rho=\underbrace{\left[\begin{array}{ccc}
\sin \left(\beta_{1}\right) & \cos \left(\beta_{1}\right) & 1 \\
\sin \left(\beta_{2}\right) & \cos \left(\beta_{2}\right) & 1 \\
\vdots & \vdots & \vdots \\
\vdots & \vdots & \vdots \\
\sin \left(\beta_{n}\right) & \cos \left(\beta_{n}\right) & 1
\end{array}\right]}_{A}\left[\begin{array}{c}
\Delta x \\
\Delta y \\
\Delta b
\end{array}\right]
$$

where $\beta_{n}$ are bearings of the stations from the receiver. The differences $\Delta \mathrm{x}, \Delta \mathrm{y}$ and $\Delta \mathrm{b}$ represent corrections (expressed in meters) to be applied to the current position and receiver clock bias estimates. If the system contains more than three stations, we can get the solution by using equation given by:

$$
\Delta p=\left(A^{T} A\right)^{-1} A^{T} \Delta \rho
$$

\section{Factors Affecting Accuracy of Position}

The accuracy of position fix is mainly determined by three factors:

- Geometry of the transmitter stations in view

- Accuracy of signal Time-of-Arrival (ToA) measurements.

- Accuracy of the ToA to range conversion.

\subsection{Geometry of the Transmitter Stations in View}

The locations of transmitter stations are generally selected to give better geometric configuration. The effect of transmitter geometry on the accuracy is usually described by Horizontal Dilution of Precision (HDOP) factor. From equation (4), the variance matrix of the position error, assuming zero mean of both position and measurement errors $E\{\Delta p\}=0$ and $\mathrm{E}\{\Delta \rho\}=0$, can be written as:

$$
\operatorname{var}[\Delta p]=\left(A^{T} A\right)^{-1} A^{-1} \operatorname{var}[\Delta \rho] A\left(A^{T} A\right)^{-1}
$$


Assuming the pseudo range measurement errors are mutually uncorrelated and have equal variance $\sigma_{\rho}^{2}$.

$$
\operatorname{var}[\Delta \rho]=\sigma_{\rho}^{2} I
$$

Then, the variance matrix of position error can be reduced to

$$
\operatorname{var}[\Delta \rho]=\left(\begin{array}{ccc}
\sigma_{x}^{2} & \sigma_{x y} & \sigma_{x b} \\
\sigma_{x y} & \sigma_{y} & \sigma_{y b} \\
\sigma_{x b} & \sigma_{y b} & \sigma_{b}^{2}
\end{array}\right)=\sigma_{\rho}^{2} \underbrace{\left(A^{T} A\right)^{-1}}_{G}
$$

where $\mathrm{G}$ part is dimensionless and depends only on the transmitter geometry.

Position accuracy using Distance Root-Mean-Square (DRMS) can be determined as a product of the measurement error $\sigma_{\rho}$, and a dimensionless multiplier dependent only on the transmitterreceiver geometry. Hence, we can extract the HDOP from the following equation:

$$
\alpha_{D R M S}=\sqrt{\sigma_{x}^{2}+\sigma_{y}^{2}}=\sigma_{\rho} \underbrace{\sqrt{G_{1,1}+G_{2,2}}}_{H D O P}
$$

\subsection{Accuracy of Signal Time-of-Arrival (ToA) Measurements}

Measuring signal ToA in eLoran receiver is made in two stages [3]. Firstly, coarse measurements are made based on the shape of the leading edge of the eLoran pulse as it provides an approximate value of TOA. Secondly, fine measurements which use the phase of eLoran signal to give an accurate value of TOA. Signal to noise ratio (SNR) of the received eLoran signal strongly determines the accuracy of TOA measurement. As eLoran signal is in the ground wave band, many error factors are compromising the noise and interference effects. These factors include the atmospheric noise, the cross-rate interference (CRI), sky wave interference, continuous-wave interference (CWI), and transmitter timing jitter and transmitting antenna impedance variations [3]. Sky wave interference, CRI and CWI are effectively mitigated by receiver processing algorithms but the residual errors from these sources may still be present in certain conditions. The calculation of SNR requires knowledge of the signal strength of the eLoran signal and the level of external noise at the same location. Moreover, ground waves are attenuated at a rate that depends on the conductivity of the surface over which they propagate. The lower the surface conductivity, the higher the rate of attenuation will be. Figure (1) shows a set of field strength curves applicable in the eLoran frequency band as a function of distance to the transmitter over different surfaces [4]. Millington method is a semi-empirical approach that can be used for determining the field strength when waves are propagating over mixed paths [4].

The characteristics of the atmospheric noise vary with location on the globe, season of the year, and time of the day. A great number of statistical models for atmospheric noise were presented in the past. The International Telecommunication Union (ITU) model consider the traditional approach to atmospheric noise modeling for eLoran uses to determine the average noise and field strengths rather than using percentile values [5]. In calculating the average annual RMS values, statistical data from all season-time blocks of the ITU model are combined in an attempt to obtain values representative of average noise conditions during the year. SNR at eLoran receiver is defined as the ratio of the eLoran signal level at the Standard Sampling Point (SSP) to the RMS level of simulated noise. The atmospheric noise produces uncorrelated ToA 
measurement errors which can be suppressed by integrating a certain number of received pulses, in an integration time $\left(T_{i}\right)$, before taking measurements [3]. This effectively increases the SNR of the received signals but at the same time it places limitations on the allowable dynamics of the user platform.

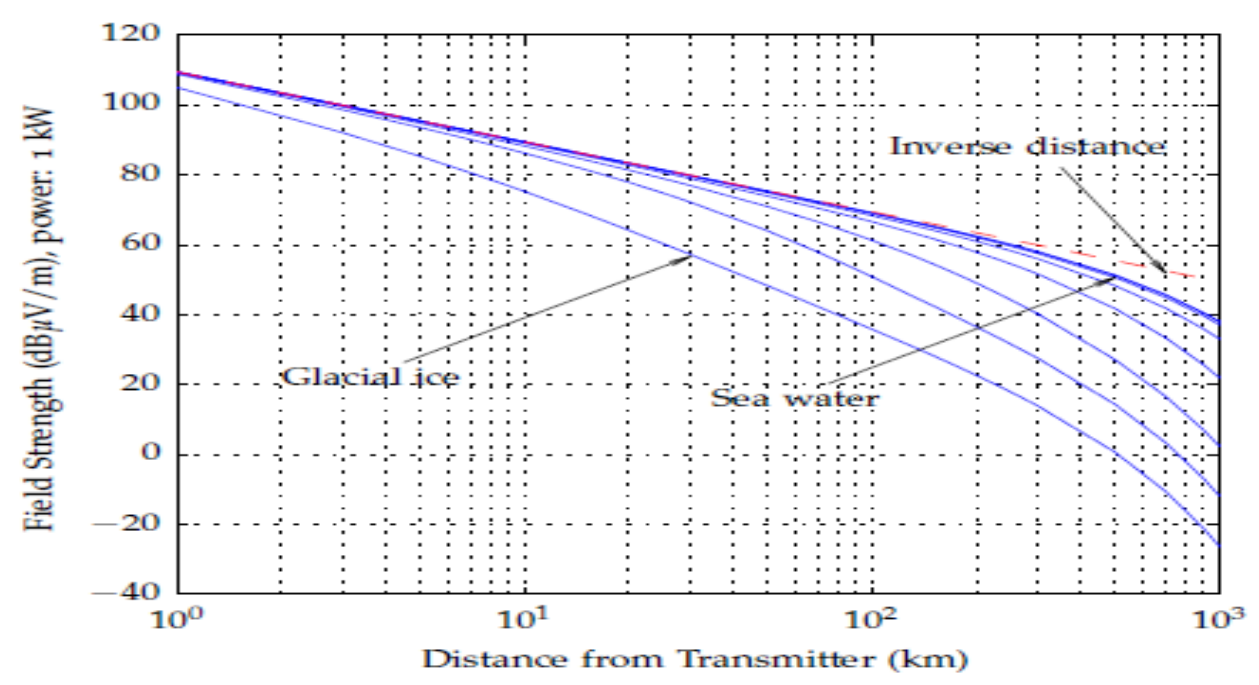

Figure (1): Ground wave field strength as a function of distance for a $1 \mathrm{~kW}$ transmission and different surface types

Pseudorange measurement error in meters can be expressed as a function of SNR in the following equation. The main driver Pseudorange of variance is the signal-to-noise ratio (SNR) of the received signal because low SNR lead to high Pseudorange variance as shown in Figure (2).

$$
\operatorname{var}[\Delta \rho]=\sigma_{\rho}^{2}=\frac{337.5^{2}}{N . S N R}
$$

$N$ is the number of eLoran pulses received within the receiver integration time. Its value depends on integration time and time of Group Repetition Interval as depicted in the following equation

$$
N=\frac{8 T_{i}}{T_{G R I}}
$$

eLoran transmitters are organized in groups called chains usually consist of 3 to 5 stations. The stations periodically broadcast groups of 8 or 9 specially shaped low-frequency and high-power pulses. The interval between successive repetitions of the groups of pulses is unique to each chain and known as the Group Repetition Interval (GRI). Its value effect on pseudorange measurement error as depicted in equation (10) so we need to select value of GRI. Allowable rates of GRI were selected in the range 4000 to 9999 . GRI must be greater than minimum GRI. . To achieve this, we need to determine the smallest time difference between the reception of transmissions from stations $\mathrm{m}$ and $\mathrm{m}+1$ which known as minimum Emission Delay (ED) [6]. The minimum time between master and secondary is $\Delta \tau_{\mathrm{m}, \mathrm{s}(\mathrm{min})}=10900 \mu \mathrm{s}$. Minimum time between secondary $\mathrm{m}$ and $\mathrm{m}+1$ is $\Delta \tau_{\mathrm{m}, \mathrm{s}(\mathrm{min})}=9900 \mu$ s and the minimum separation between the last secondary transmission and master transmission from the next GRI is $\Delta \tau_{\mathrm{m}, \mathrm{s}(\mathrm{min})}=9900 \mu \mathrm{s}$. The minimum GRI can be determine by the following equation: 


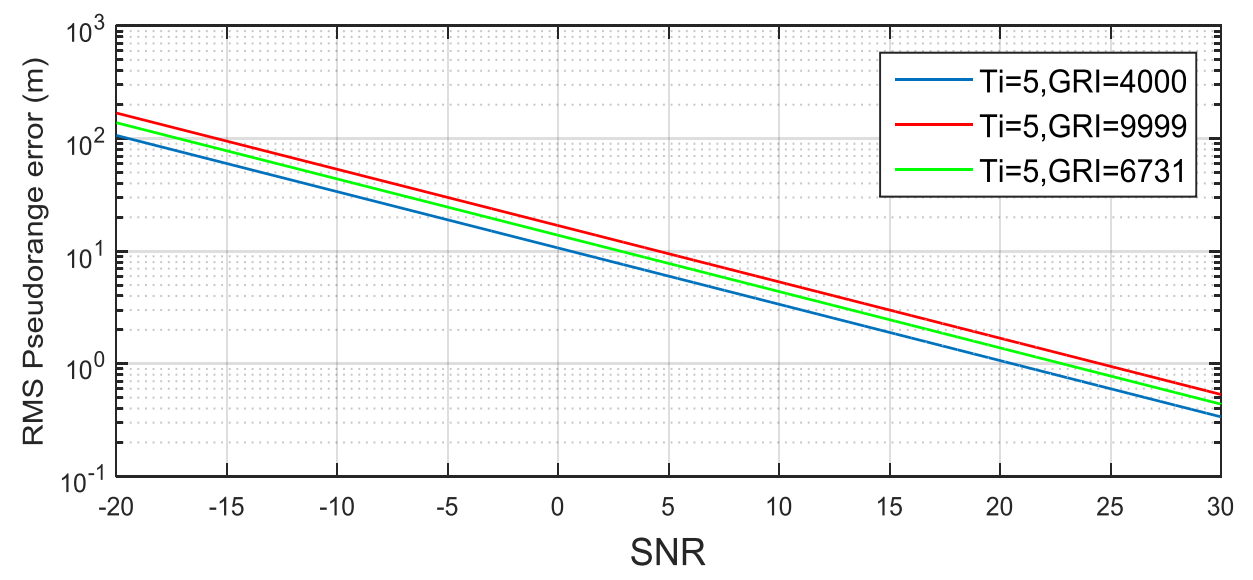

Figure (2): RMS pseudorange error due to AWGN as a function of SNR for different GRI

$$
T_{G R I, \text { min }}=\sum_{m=1}^{N_{s t}} \Delta \tau_{E D, m}=\sum_{m=1}^{N_{s t}} \Delta \tau_{m, \text { min }}+\frac{r_{m}}{v_{\text {prop }}}
$$

where $r_{m}$ is distance between loran stations and $v_{\text {prop }}$ is speed of propagated signal.

Careful selection of GRI and transmission times ensures that stations operating in a chain do not interfere with each other (CRI) and transmitters that broadcasting close to the eLoran frequency bands (CWI) do not interfere with eLoran signal. There are another constrains such that eliminate all GRI that exceeding the maximum transmitter pulse rate $700 \mathrm{pps}$ and eliminate all GRI that are divisible by 100 and $500 \mu$ s to reduce the effect of CWI [6].

We suggest a slightly modified version of equation (9), [3]:

$$
\operatorname{var}[\Delta \rho]=\sigma_{\rho}^{2}=\frac{337.5^{2}}{N . S N R}+\frac{c_{1}}{N}+c_{2}
$$

$c_{1}$ accounts for transmitter related noise, which is assumed to be $c_{1}=36 \mathrm{~m}^{2}, \mathrm{~N}$ is the number of pulses used in signal integration, $c_{2}$ accounts for other sources of variation in the pseudorange measurements $c_{2}=12 \mathrm{~m}^{2}$.

\subsection{Accuracy of the ToA to range conversion}

The ground wave accumulates a delay compared to the expected propagation time over the same distance in free space. This delay is the accumulation of three components or factors:

Primary Factor (PF): The Primary Factor delay is the difference between propagation of the signal in the earth's atmosphere as opposed to in free space. Due to the refractive index of the atmosphere $\left(\mathrm{n}_{P F}=1.000338\right)$, the PF speed of the Loran signal through the atmosphere is given by $\mathrm{c}_{P F}=299,691,162 \mathrm{~m} / \mathrm{s}$

Secondary Factor $(S F)$ : In addition to propagating through the atmosphere, a significant proportion of the Loran signal wave-front will penetrate the surface of the Earth. Due to the dielectric properties of the surface this part of the signal will travel more slowly than in the atmosphere. As the electrical conductivity of the surface decreases a greater proportion of the signal will penetrate the ground and the wave-front will propagate more slowly. The best conducting surface is seawater (which has a nominal conductivity of $5 \mathrm{~S} / \mathrm{m}$ ). To model and account for the SF using the equations described by P. Brunavs [7], as shown in Figure (3). 


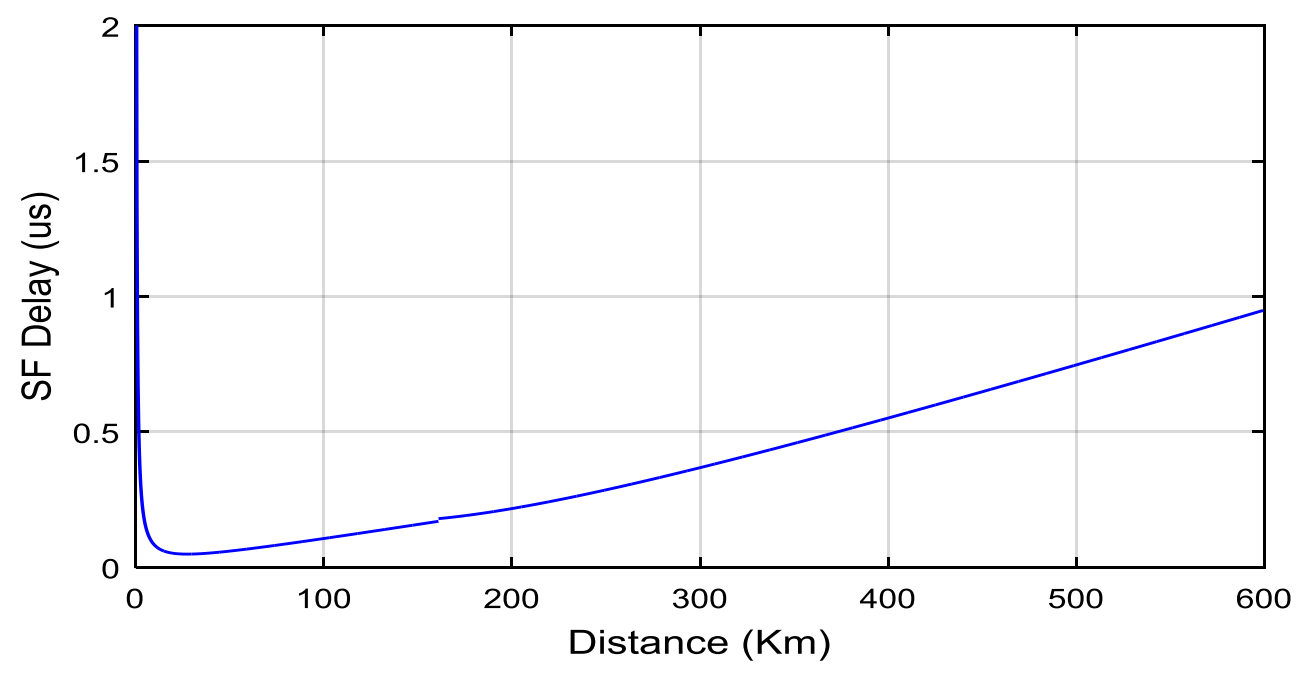

Figure (3): Secondary Factor Delay from Brunaves' equation

Additional Secondary Factor (ASF): Any land encountered with a surface conductivity lower than seawater will delay the signal even further. This additional delay is known as Additional Secondary Factor or ASF. To account for land propagation, the receiver must be provided with built-in tables of ASF values which would be measured and published by the service provider. A value for ASF is required for each transmitter, and at each location at which the receiver is to be used. In Figure (4), the expected delay for dry land and seawater.

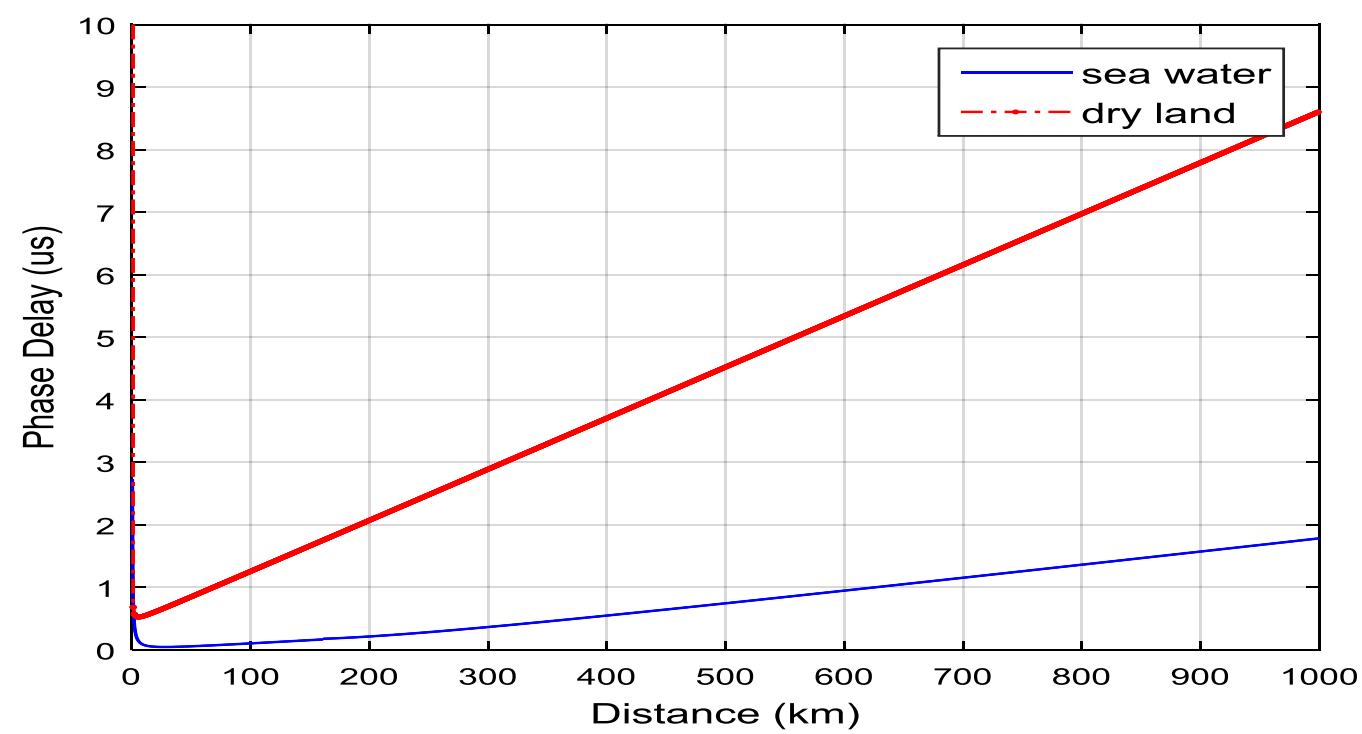

Figure (4): Phase delay of dry land and sea water

The ASF delay is the total cumulative delay the signal experiences of sections with different ground conductivity. There exist a number of techniques to predict the delay, relative to the speed of light, of a signal propagating along a path of given conductivity [8], [9]. A method developed by Millington involves dividing the path into small sections of constant conductivity, and computing the phase delay for each section in turn. Modeling ASF using this method requires a detailed and accurate database of ground conductivity. 
The current method for providing ASF data for a limited geographical area say a harbor approach by comprehensive measurement campaign of the area. The surveyed data is then uploaded to the user's receiver. The proposed data format is a 'grid' of spot values of ASF at regular spacing throughout the harbor and approach channel area for each Loran transmitter. Experimental work in the USA, [10] has indicated that 500m grid spacing for the values is an optimal figure to be able to describe typical spatial variations accurately. For ease of use the grid lines follow the WGS84 lines of Latitude and Longitude. In latitude, we use 0.005 degree spacing and the longitudinal values are scaled appropriately depending on the latitude. The value for each cell is then assigned as the mean of all of the surveyed data that lies within that cell. This type of grid is currently the preferred database format implemented within the commercial eLoran receivers available. This is because the format is easy to use; the operation is as so the receiver looks up the ASF values, one per eLoran transmitter, for the cell within which it is currently located based on a sea-water only position computation. The accuracy of ASF data stored in eLoran receiver effect on Loran position by factor $\sigma_{A S F}^{2}$. Not taking ASFs into account can lead to ranging errors of up to $2 \mathrm{~km}$, [10]. The true ASF for a user in a certain area can be written as the sum of a spatial value plus a temporal term.

$$
A S F=\overline{A S F}+A S F_{\text {temporal }}
$$

Over time there will be some variation from the fixed ASF values stored in the receiver due to Seasonal effects such as the amount of rain-water soaking into the soil, or the formation of ice in the winter will change the electrical conductivity of the land. In addition, changes in the temperature, pressure or moisture content of the atmosphere will alter the PF speed of light. It is known as temporal variations. Its effect appears on loran position accuracy in terms $\delta_{\text {temp }}^{2}, \sigma_{\text {temp }}^{2}$ which represent bias and variance in temporal variation respectively. So we can represent Pseudorange measurement error in meters.

$$
\operatorname{Var}[\Delta \rho]=\sigma_{\rho}^{2}=\underbrace{\frac{337.5^{2}}{N . S N R}+\frac{c_{1}}{N}}_{\text {ToAmeasurement }}+\underbrace{\sigma_{A S F}^{2}+\delta_{\text {temp }}^{2}+\sigma_{\text {temp }}^{2}}_{\text {ToA to Range conversion }}+\sigma_{\text {noise }}^{2}
$$

\section{Measurement of eLoran Positioning Accuracy}

There are numerous ways of measuring the accuracy of positioning systems. The purpose of this section is to introduce the most commonly used accuracy measures. Since eLoran does not provide height information, this section will focus only on 2D (horizontal) accuracy measures. It will be assumed throughout that the performance is measured at a fixed location (static performance) and that the underlying position error process is stationary. We concern in this section to measure accuracy of position by using Distance Root-Mean-Square (DRMS) method, [11]. We assume eLoran receiver utilize the Weighted Least Squares (WLS) position determination algorithm. If the measurement errors are uncorrelated but have different variance, the optimum solution is given by the WLS method in equation:

$$
\Delta p=\left(A^{T} W A\right)^{-1} A^{T} W \Delta \rho
$$

where the weight matrix $W$ is given by:

$$
W=(\operatorname{var}(\Delta \rho))^{-1}
$$

The variance matrix of the position error can be obtained 


$$
\operatorname{var}(\Delta p)=\left(\begin{array}{ccc}
\sigma_{x}^{2} & \sigma_{x y} & \sigma_{x b} \\
\sigma_{x y} & \sigma_{y}^{2} & \sigma_{y b} \\
\sigma_{x b} & \sigma_{y b} & \sigma_{b}^{2}
\end{array}\right)=\underbrace{\left(A^{T} W A\right)^{-1}}_{C}
$$

The DRMS accuracy can then be expressed simply as:

$$
\alpha_{D R M S}=\sqrt{C(1,1)+C(2,2)}
$$

Position accuracy is often stated as a 2DRMS figure rather than DRMS, which is simply equal

$$
\alpha_{2 D R M S}=2 . \alpha_{D R M S}
$$

\section{Experimental Work and Performance Analysis}

As mention above, we use GLA model to establish eLoran system in Egypt. It is implemented in MATLAB where the coverage is divided into grids consisting of rectangular elements of equal sizes, typically $0.1^{\circ}$ in latitude by $0.1^{\circ}$ in longitude. At each point in the grid the individual coverage limiting factors are modeled and the resulting data arrays are stored. When required, for coverage computation, these are then loaded into memory. In accuracy module of coverage software, we select eLoran stations to include in analysis and we set up a region over which coverage is required. Total five stations can provide radio navigation service in Egypt as shown in Figure (5). The locations of these new transmitters in Table- 3 are selected based on the

\begin{tabular}{|c|c|c|c|c|}
\hline GRI & Station & Position & ED (us) & PWR (KW) \\
\hline \multirow{5}{*}{6731} & $\begin{array}{l}\text { Menia } \\
\text { (Master) }\end{array}$ & $\begin{array}{ll}27.5 & \mathrm{~N} \\
30 & \mathrm{E}\end{array}$ & 0.00 & $1000 \mathrm{KW}$ \\
\hline & $\begin{array}{c}\text { Matrouh } \\
\text { (Secondary 1) }\end{array}$ & $\begin{array}{ll}31.5 & \mathrm{~N} \\
26.2 & \mathrm{E} \\
\end{array}$ & 13000 & $1000 \mathrm{KW}$ \\
\hline & $\begin{array}{c}\text { Port-Said } \\
\text { (Secondary 2) }\end{array}$ & $\begin{array}{r}30.92511 \mathrm{~N} \\
32.671567 \mathrm{E} \\
\end{array}$ & 25000 & $1000 \mathrm{KW}$ \\
\hline & $\begin{array}{c}\text { Ash-shaykh } \\
\text { (Secondary 3) }\end{array}$ & $\begin{array}{l}28.15444 \mathrm{~N} \\
34.76126 \mathrm{E}\end{array}$ & 365000 & $1000 \mathrm{KW}$ \\
\hline & $\begin{array}{l}\text { Marsa-Alam } \\
\text { (Secondary 4) }\end{array}$ & $\begin{array}{l}24.88699 \mathrm{~N} \\
34.19199 \mathrm{E} \\
\end{array}$ & 49000 & $1000 \mathrm{KW}$ \\
\hline
\end{tabular}
coverage and accuracy simulation results.

Table 3: Egypt Loran stations

\subsection{Geometry of the Transmitter Stations in View}

The location of transmitter stations is selected to give better geometric configuration, but it is almost impossible to secure those sites to achieve the best coverage. As mention previous, the geometry of transmitter station can be represented by HDOP as shown in Figure (6).

\subsection{Accuracy of Signal Time-of-Arrival (ToA) Measurements}

The level of accuracy is highly dependent on the variance of the Pseudorange values. The high value of the pseudorange variance leads to poor position accuracy. The main driver of pseudorange variance is SNR. To calculate SNR, we need to determine signal strength of Loran signal and the level of noise at the same location. In GLAs' coverage prediction software, ground wave field strength is calculated using propagation curves for different ground conductivities at $100 \mathrm{kHz}$ as shown in previous section. In this study, ground wave field strengths will be modeled using the Millington's method as described in the ITU-R 
Recommendation [4]. The signal strength of two Loran stations is shown in Figure (7) and Figure (8).

As mentioned above, the dominant noise source in the Loran band is atmospheric noise. Atmospheric noise is computed at different percentiles and generated based on the model presented in ITU Recommendation P372-9[5]. Figure (9) show the distribution of the predicted average daytime noise over Egypt.

From previous results, we can calculate SNR of each station and determine the pseudorange variance error. In Figure (10), we show the SNR of eLoran station. An accurate estimate of pseudorange measurement variance under the assumption of white noise occur if the range of SNR from $-10 \mathrm{~dB}$ to $+40 \mathrm{~dB}$.

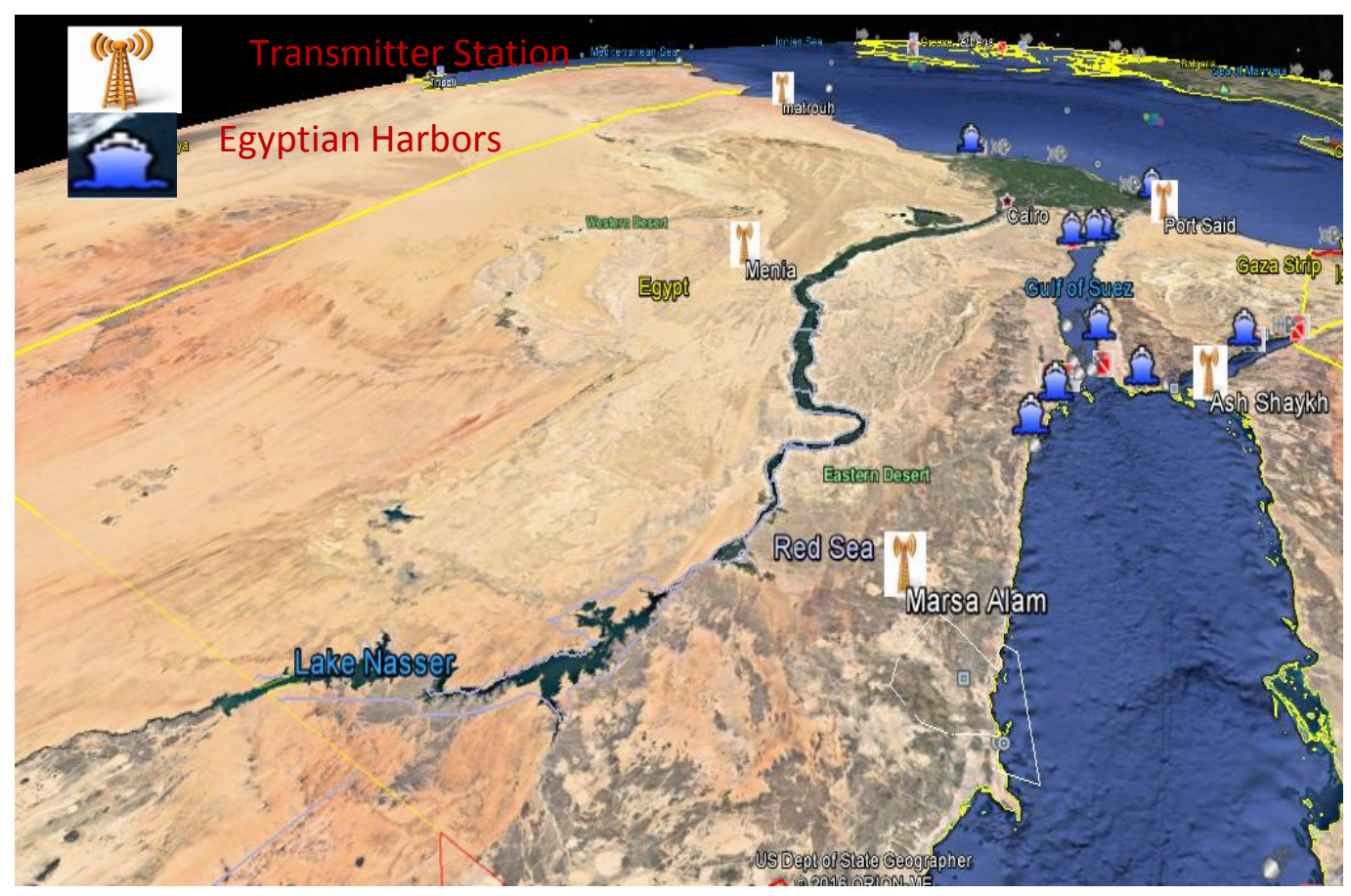

Figure (5): Selected locations for the eLoran transmitter station and major harbors

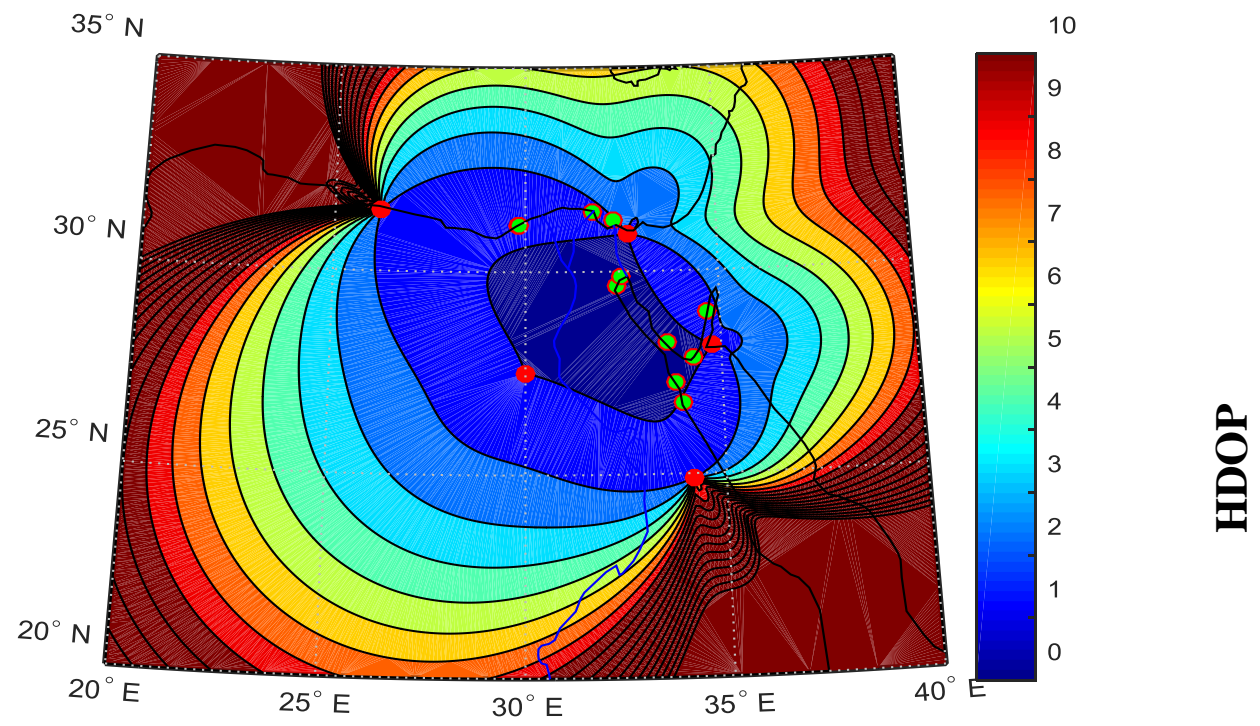

Figure (6): HDOP distribution over Egypt using Loran transmitter stations 


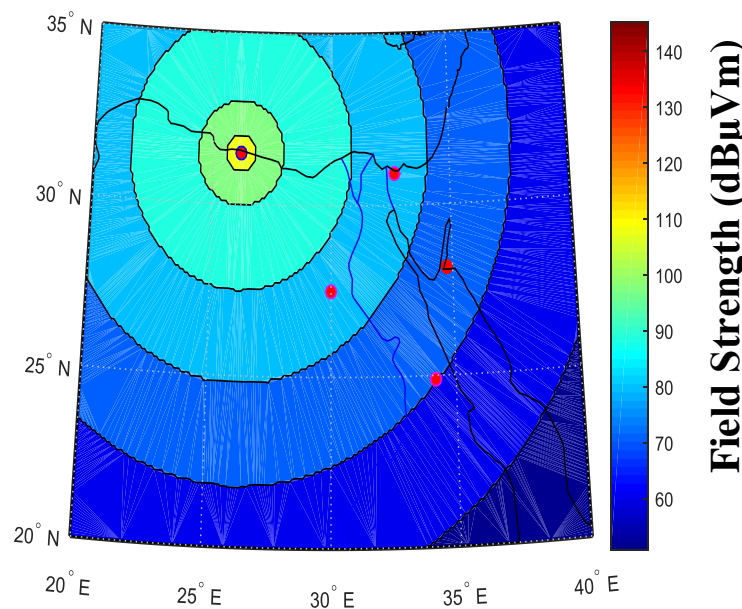

Figure (7): Predicted ground wave field strength for the Matrouh station

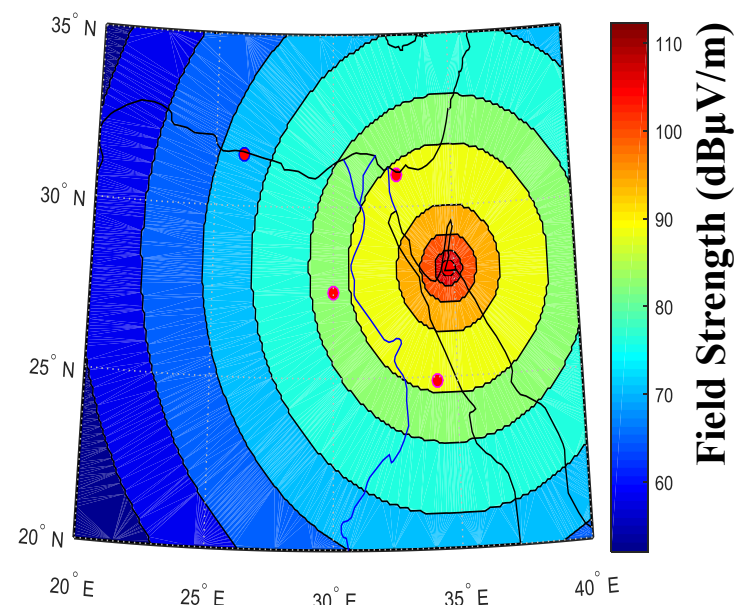

Figure (8): Predicted ground wave field strength for the Ash Shaykh station

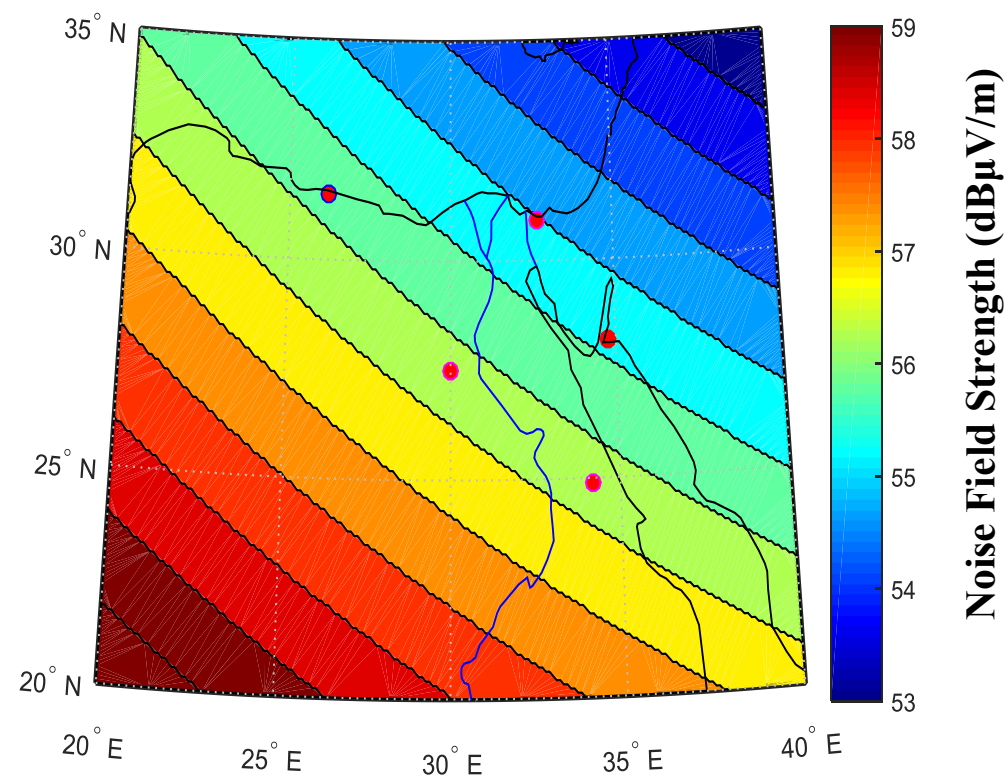

Figure (9): Average daytime atmospheric noise over Egypt

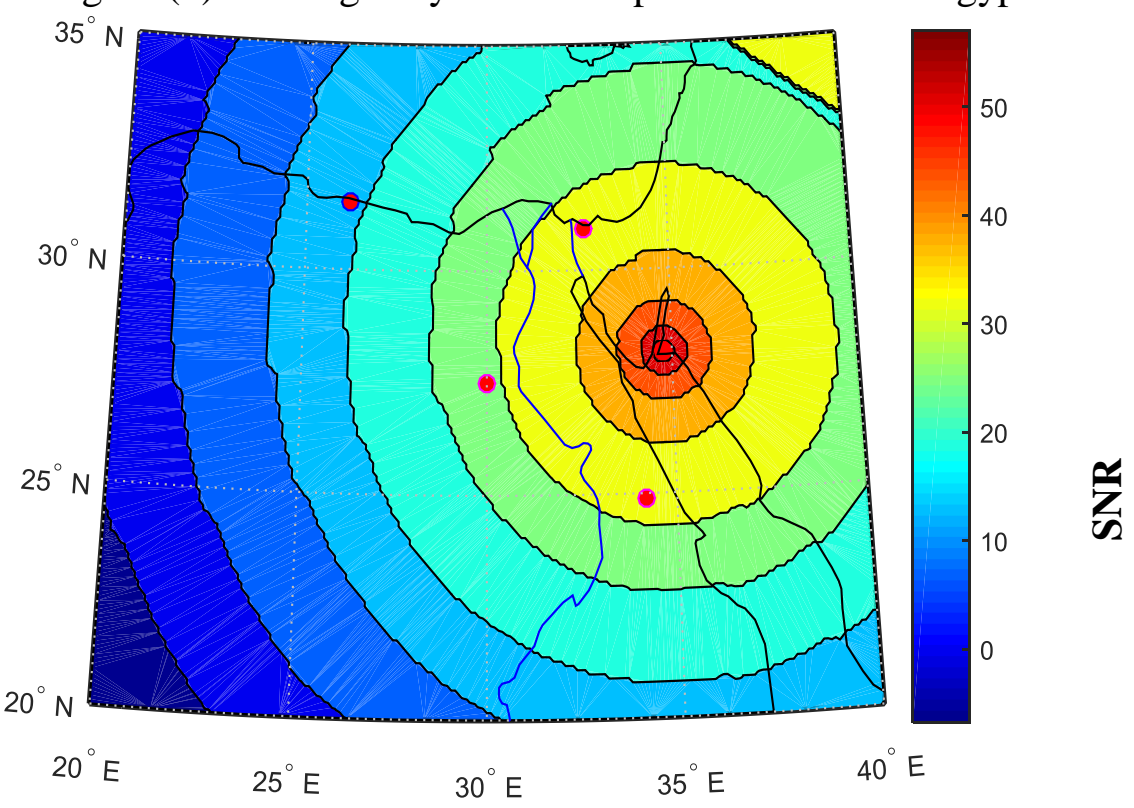

Figure (10): Predicted SNR for the Ash-Shaykh eLoran station 


\subsection{GRI Selection}

As mention above, the interval between successive repetitions of the groups of pulses is unique to each chain and known as GRI. Its value effects on pseudorange measurement error as shown in equation (10). So GRI value can be set by the following steps:

i. GRI range of 4000 to 9999 (tens of $\mu s$ )

ii. Determine minimum GRI where the minimum value from Table-4 is $\left(T_{G R I, \min }=58643 \mu s\right)$.

Table 4: Minimum GRI value

\begin{tabular}{lll|c}
\hline \hline Stations & & $\begin{array}{c}\text { Difference in ED } \\
\Delta \tau_{E D, m}(\mu s)\end{array}$ \\
\hline \hline Menia $\longrightarrow$ & Matrouh & 12824 \\
\hline Matrouh $\longrightarrow$ & Port-Said & 11963 \\
\hline Port-Said $\longrightarrow$ & Ash-shaykh & 11130 \\
\hline Ash-shaykh $\longrightarrow$ & Marsa-Alam & 11128 \\
\hline Marsa-Alam $\longrightarrow$ & Menia & 11598 \\
\hline \hline \multicolumn{1}{c|}{ Sum } & 58643 \\
\hline \hline
\end{tabular}

iii. Determine which Loran stations will interfere with the proposed chain as show in Figure (11) and close up of it in Figure (12).

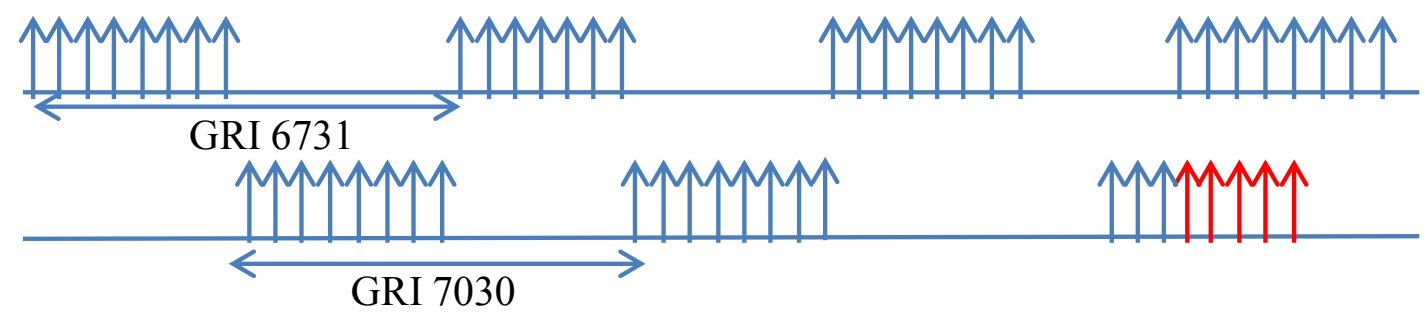

Figure (11): Cross-rate interference

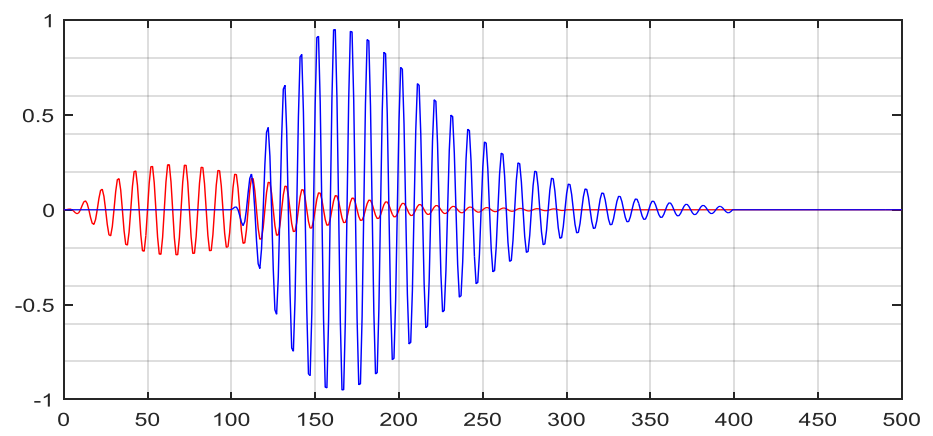

Figure (12): Close up of cross-rate interference

iv. The important parameter measurement is Crossover Time which corresponding to number of successive groups of pulses affected by overlaps between two GRI.

v. The shape of Loran pulses in Figure (13) show that $99 \%$ the signal's energy is concentrated between 90 and $110 \mathrm{kHz}$. CWI result from non-Loran transmitters operate near to loran band and these interfere to greater or lesser depending on GRI. The receiver Susceptibility to CWI depends on the particular GRI of interest. In Figure (14), Loran spectrum reveals distinct spectral lines every $1 /(2 * \mathrm{GRI})$ and CWI appear as single lines. If CWI fall between the spectral lines of Loran, they less harmful than they coincide. 

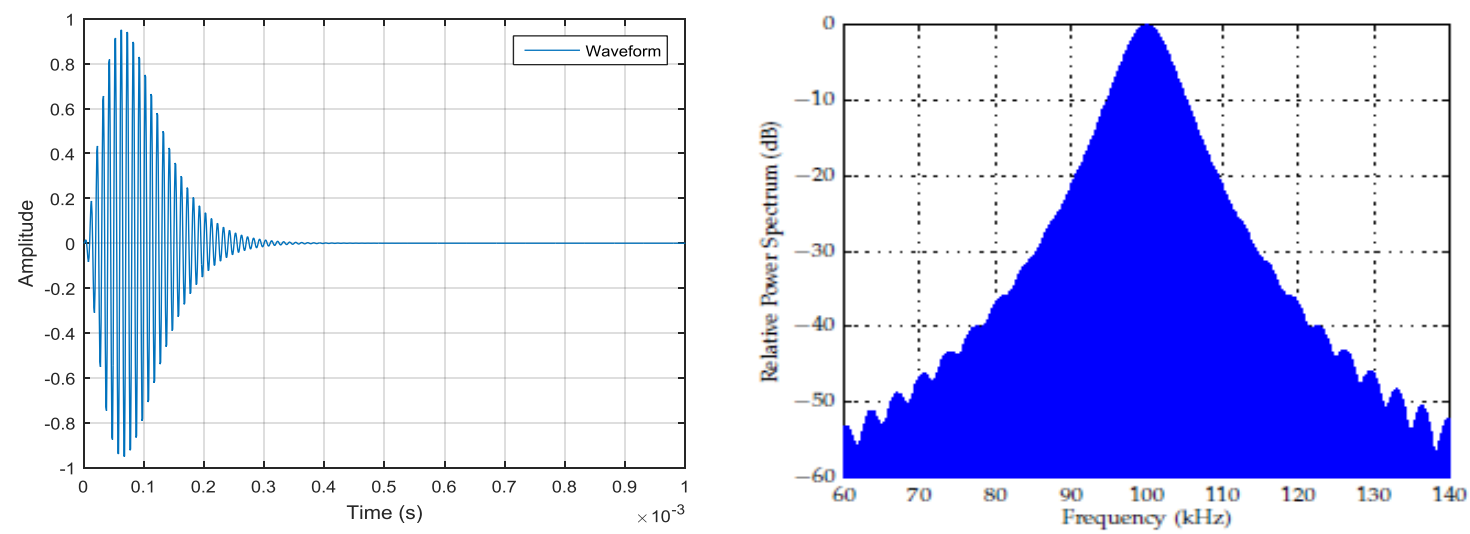

Figure (13): Loran pulse and power spectrum
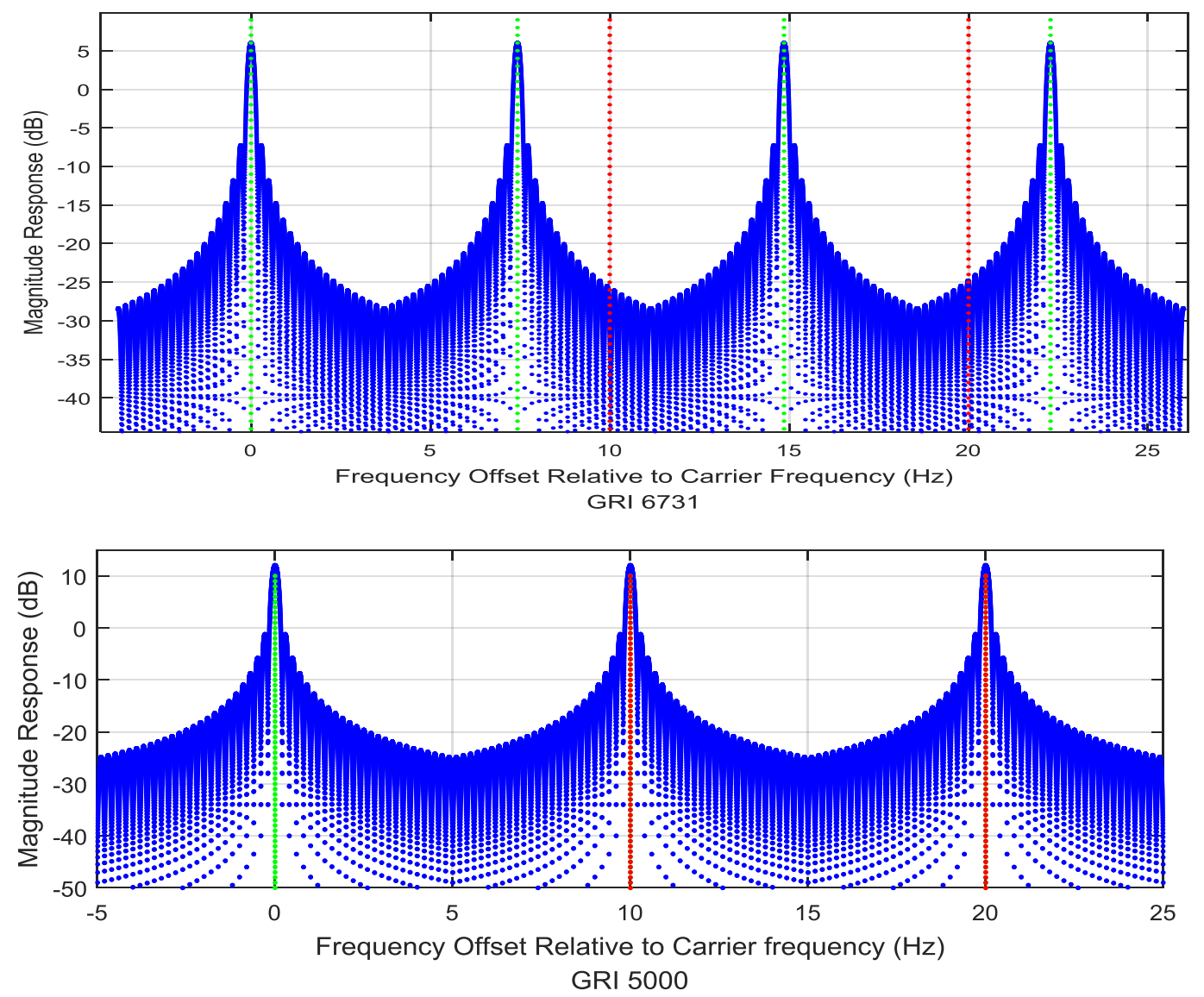

Figure (14): Detail of Loran spectrum for two different GRI values; continuous- wave interference is depicted as res lines

vi. Use the minimum GRI and the list of stations that may interfere to obtain a list of GRIs that are relatively prime to all GRIs that may interfere with the proposed chain.

vii. Eliminate all GRIs that would result in exceeding the maximum transmitter pulse rate 700 $\mathrm{p} / \mathrm{s}$.

viii. The need to space into GRI for presence of signal from Loran simulators 
The overall procedure of GRI selection is summarized in Figure (15)

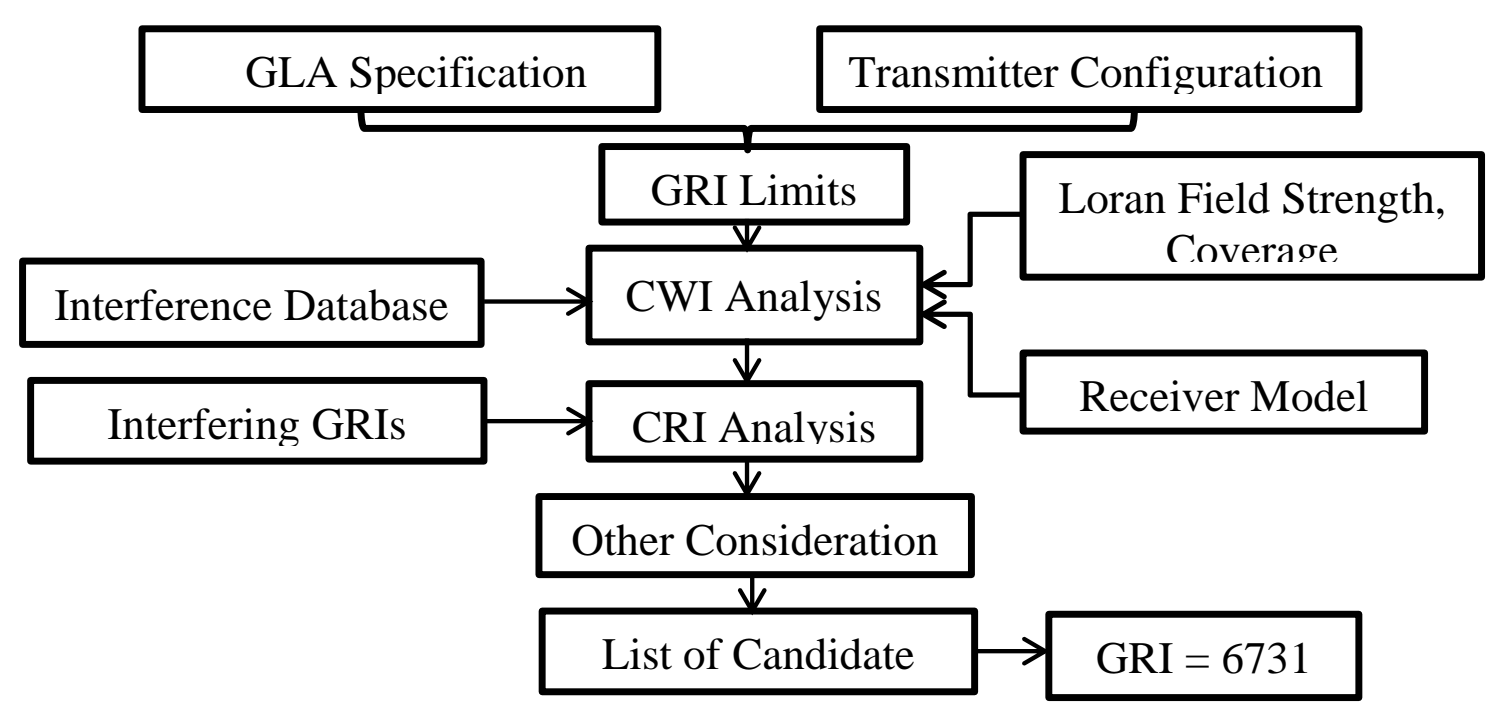

Figure (15): The overall procedure of GRI selection

\subsection{ASF Maps for Spatial Error Mitigation}

As mention in previous section, the ASF is the largest error source in eLoran. The spatial variations of ASF cannot be effectively mitigated by the differential eLoran corrections so that ASF maps are utilized. Once spatial ASF variations are surveyed over a region, eLoran receivers store the spatial ASF variation maps and apply the information as spatial corrections. ASF maps with the grid size of $500 \mathrm{~m}$ are generally acceptable for maritime users. Figures (16) through (19) show the predicted ASF values for different harbors and Suez Canal.
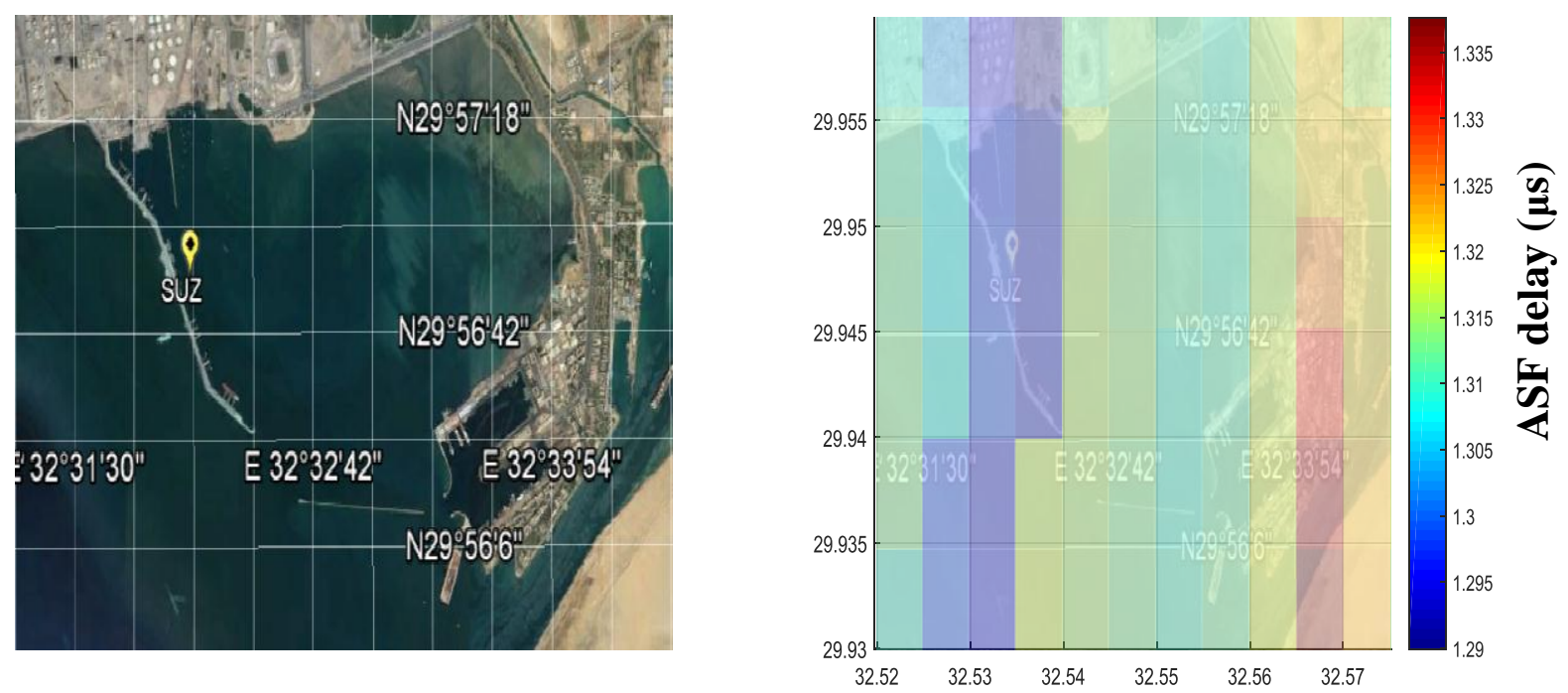

Figure (16): Port Said Basic ASF grid of the data at 500m spacing measured at Suez port 

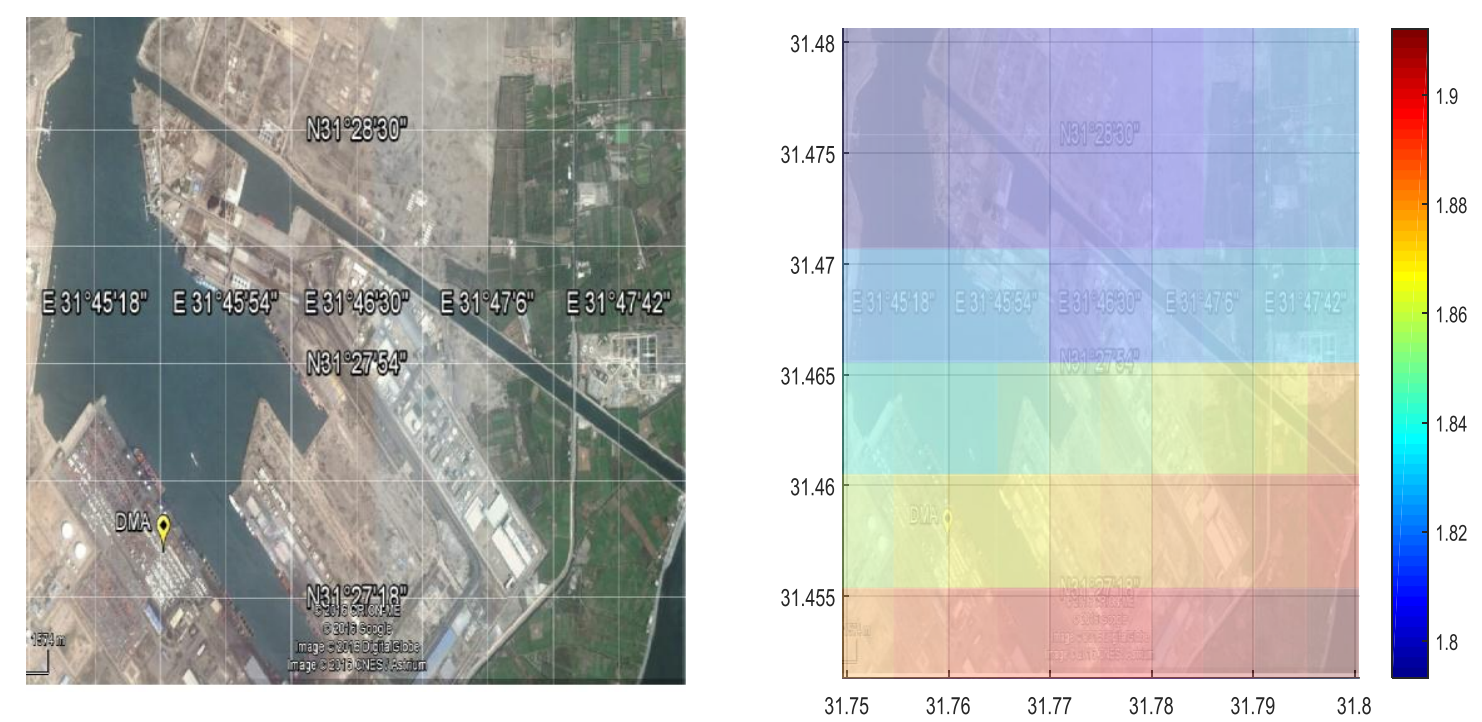

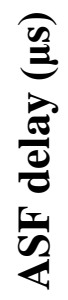

Figure (17): Port Said Basic ASF grid of the data at 500m spacing measured at Sharq Port said
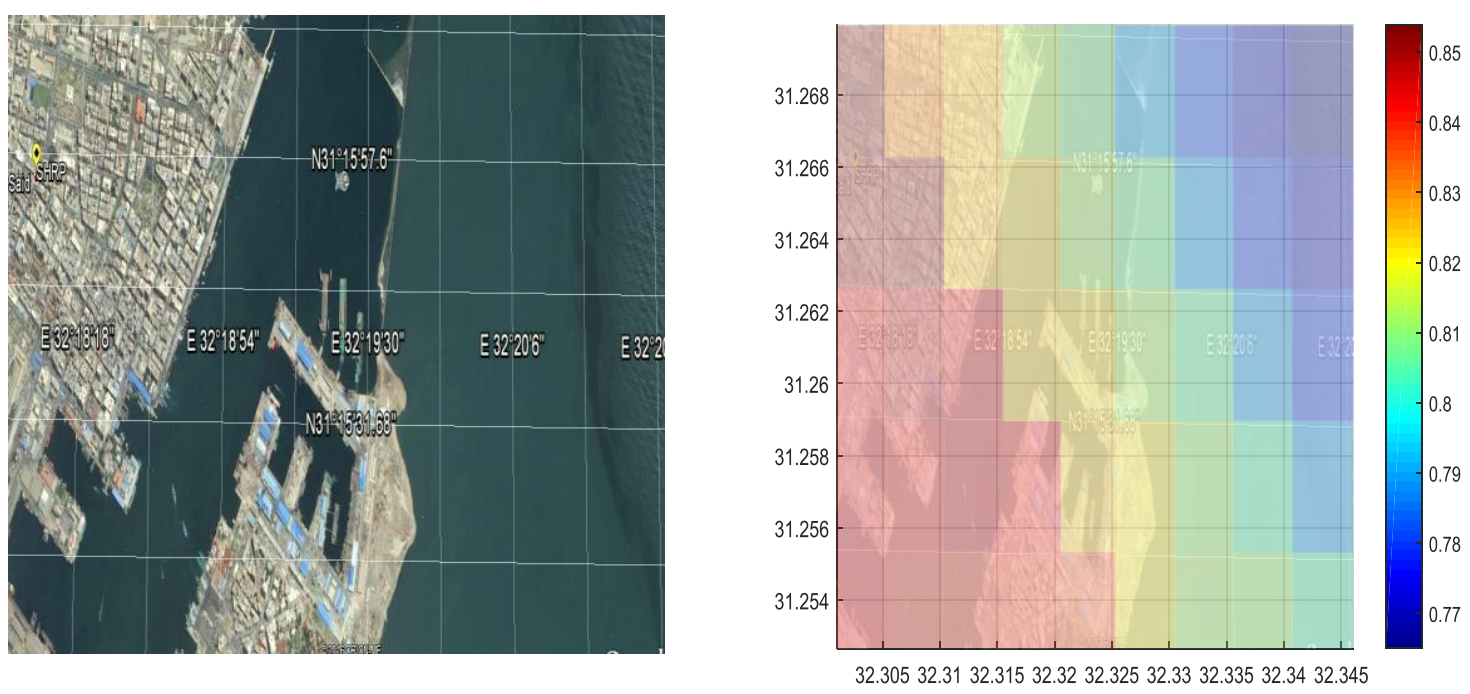

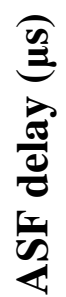

Figure (18): Port Said Basic ASF grid of the data at $500 \mathrm{~m}$ spacing measured at Domiat port

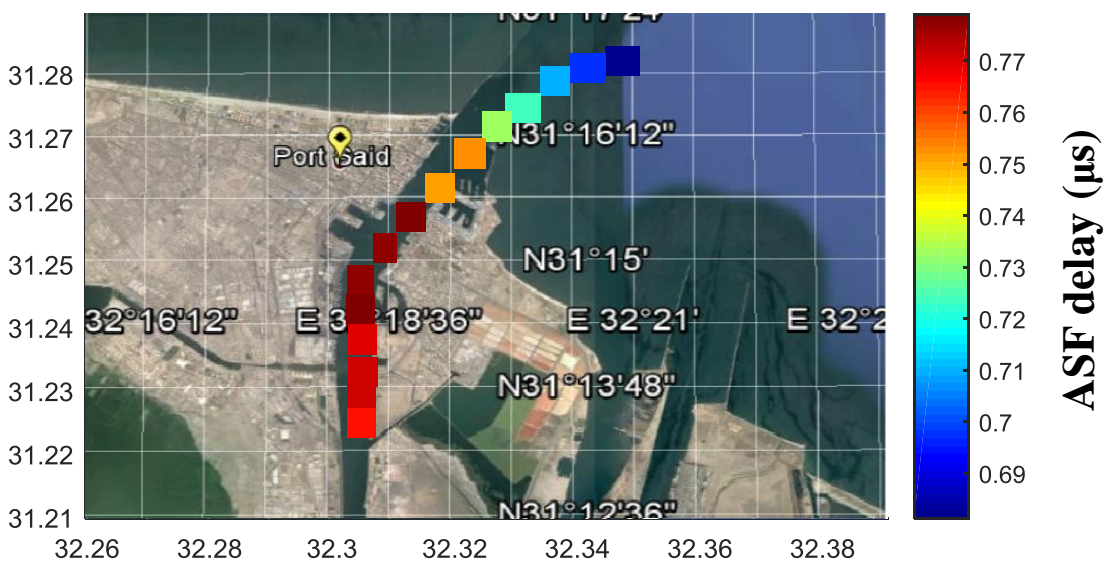

Figure (19): Port Said Basic ASF grid of the data at 500m spacing measured for surveyed path 
When all the data arrays are available, algorithms within the software then test each grid point to see whether the eLoran signals meet certain acceptance criteria. With data at each point on the map, the accuracy is calculated and plotted as shown in Figure (20).

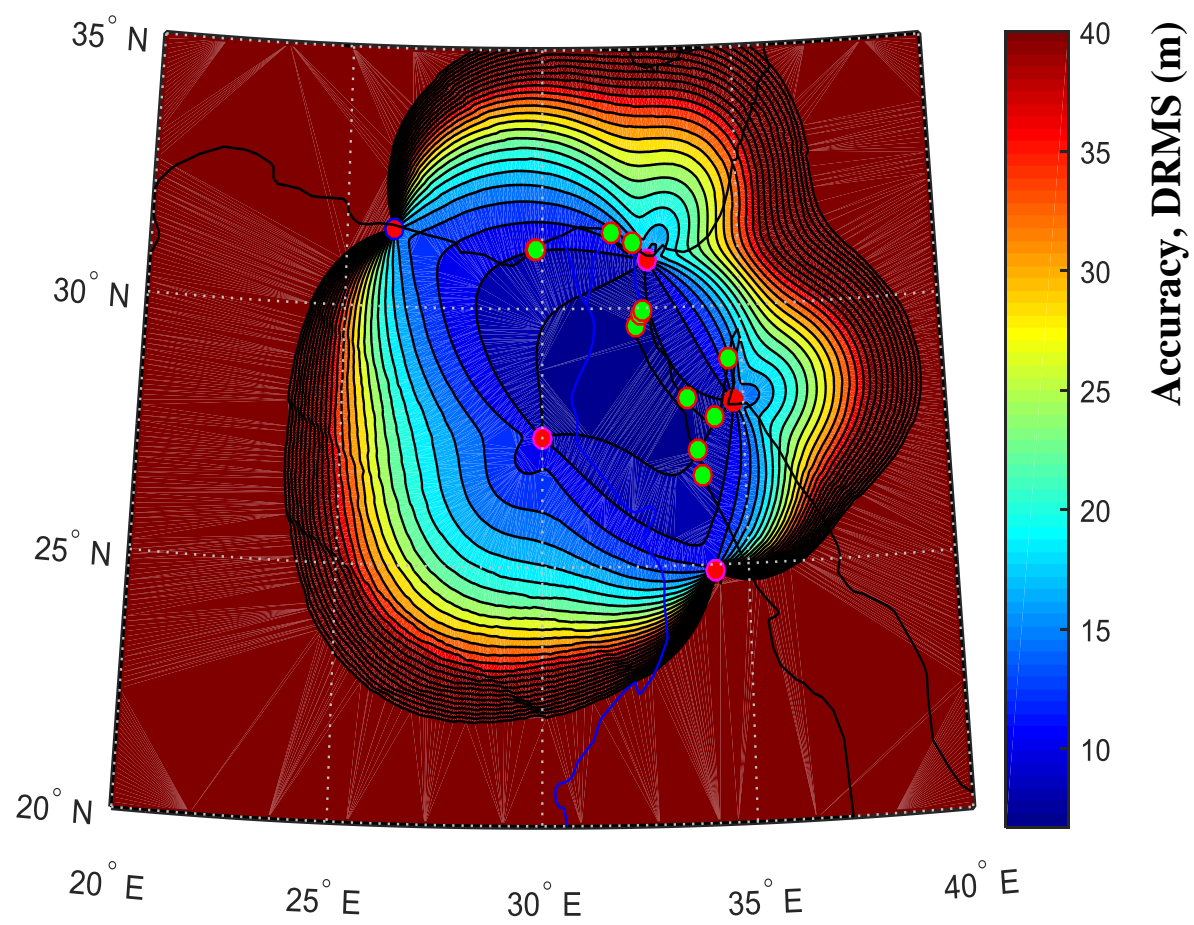

Figure (20): Predicted position accuracy of proposed eLoran chain

\section{Conclusion and Future Work}

In this paper we presented a simulation-based proposal to establish the eLoran navigation system for the Egyptian marine sector according to its positioning accuracy. We have generated repeatable accuracy plots for Egypt by installation of eLoran stations at candidate locations. The simulation results for this proposal of Egyptian eLoran achieved the IMO's accuracy requirement of better than 20m over most of Egyptian's coastal borders and harbors. During this study, we assumed that perfect ASFs were provided to the user, so that any biases in the measurements were eliminated. The future work will be intended into studying the other factors affecting on the eLoran performance as a positioning system for Egyptian harbors including availability, continuity and integrity.

\section{References}

[1] Grant A., Williams P., Ward N., and Basker S., GPS Jamming and the Impact on Maritime Navigation, The Journal of Navigation, 2009, Vol. 62, pp. 173-187.

[2] International Loran Association, Enhanced Loran (eLoran) Definition Document, 2007. v. 1.0 .

[3] Jan Šafár: Analysis, Modelling and Mitigation of Cross-Rate Interference in Enhanced Loran, Ph.D thesis, Czech Technical University in Prague, August 2014.

[4] International Telecommunication Union, ITU-R Rec. 368-9, Ground-Wave Propagation Curves for Frequencies between $10 \mathrm{kHz}$ and $30 \mathrm{MHz}, 2007$.

[5] International Telecommunication Union, ITU-R P.372-9, Radio Noise, 2007.

[6] R. W. Nutting, Loran-C Rate Selection Process. United States Coast Guard, 1988. 
[7] Brunavs P., Phase lags of $100 \mathrm{kHz}$ radio frequency ground wave and approximate formulas for computation, 1997.

[8] Millington, G, "Ground-wave Propagation over an Inhomogeneous Smooth Earth", Proceedings of the IEE, Series 96, Number 3, 1949

[9] Last, J.D., Williams, P., Peterson, B. and Dykstra, K., 'Propagation of Loran-C Signals in Irregular Terrain - Modelling and Measurements Part 1: Modelling', 29th Annual Convention and Technical Symposium, International Loran Association, Washington DC, Washington USA, 13-15 November 2000.

[10] Williams, P. \& Last, J.D., 'Mapping the ASFs of the Northwest European Loran-C system', Journal of the Royal Institute of Navigation, 53, 2, pp225-235, May 2000 (Invited).

[11] G. Johnson, K. Dykstra, C. Oates, et al. "Navigating harbors at high accuracy without GPS: eLoran proof-of-concept on the Thames River," Proc. ION National Technical Meeting, San Diego, CA, 22-24 January 2007. 